\title{
Effect of Virtual Reality on Cognition in Stroke Patients
}

\author{
Bo Ryun Kim, M.D. ${ }^{1}$, Min Ho Chun, M.D., Lee Suk Kim, M.D., Ji Young Park, M.D. ${ }^{2}$ \\ Department of Rehabilitation Medicine, Asan Medical Center, University of Ulsan College of Medicine, Seoul 138-736, \\ ${ }^{1}$ Jeju National University College of Medicine, Jeju 690-767, ${ }^{2}$ Philip Hospital, Seoul 138-170, Korea
}

Objective To investigate the effect of virtual reality on the recovery of cognitive impairment in stroke patients. Method Twenty-eight patients (11 males and 17 females, mean age 64.2) with cognitive impairment following stroke were recruited for this study. All patients were randomly assigned to one of two groups, the virtual reality (VR) group ( $\mathrm{n}=15)$ or the control group $(\mathrm{n}=13)$. The VR group received both virtual reality training and computerbased cognitive rehabilitation, whereas the control group received only computer-based cognitive rehabilitation. To measure, activity of daily living cognitive and motor functions, the following assessment tools were used: computerized neuropsychological test and the Tower of London (TOL) test for cognitive function assessment, Korean-Modified Barthel index (K-MBI) for functional status evaluation, and the motricity index (MI) for motor function assessment. All recruited patients underwent these evaluations before rehabilitation and four weeks after rehabilitation.

Results The VR group showed significant improvement in the K-MMSE, visual and auditory continuous performance tests (CPT), forward digit span test (DST), forward and backward visual span tests (VST), visual and verbal learning tests, TOL, K-MBI, and MI scores, while the control group showed significant improvement in the K-MMSE, forward DST, visual and verbal learning tests, trail-making test-type A, TOL, K-MBI, and MI scores after rehabilitation. The changes in the visual CPT and backward VST in the VR group after rehabilitation were significantly higher than those in the control group.

Conclusion Our findings suggest that virtual reality training combined with computer-based cognitive rehabilitation may be of additional benefit for treating cognitive impairment in stroke patients.

Key Words Stroke, Cognition, Cognitive training, Virtual reality

Received September 29, 2010; Accepted February 5, 2011

Corresponding author: Min Ho Chun

Department of Rehabilitation Medicine, Asan Medical Center, University of Ulsan College of Medicine, 388-1, Pungnap-2 dong, Songpa-gu, Seoul 138-736, Korea

Tel: +82-2-3010-3800, Fax: +82-2-3010-6964, E-mail: mhchun@amc. seoul.kr

(c) This is an open-access article distributed under the terms of the Creative Commons Attribution Non-Commercial License (http://creativecommons.org/ licenses/by-nc/3.0) which permits unrestricted noncommercial use, distribution, and reproduction in any medium, provided the original work is properly cited. Copyright $\odot 2011$ by Korean Academy of Rehabilitation Medicine

\section{INTRODUCTION}

Cognitive impairment after stroke is an important factor affecting independent function and activity participation of patients, and dementia syndrome has been known to occur in approximately 8 to $26 \%$ of stroke patients within 12 months of the onset of stroke. ${ }^{1,2}$ In cases of severe cognitive impairment, it decreases morale and motivation to participate in an intensive rehabilitation program during the acute phase. It may also affect the 
ability to acquire motor skills, thus becoming the main cause of poor prognosis for rehabilitation. In addition, it may affect only one area of cognitive function, but in most cases, it is accompanied by impairment of the overall area of attention, concentration, memory, spatial processing skills, language, problem-solving skills, and planning skills. Therefore, accurate assessment of cognitive function and prompt initiation of treatment in the early stage is needed for successful rehabilitation..$^{3-5}$

In the meantime, variable cognitive rehabilitation programs using computer technology have been developed for cognitive rehabilitation, and several studies on braininjured patients have reported that computer-assisted cognitive therapy can be effective in treating cognitive disorders of various areas including memory, attention, spatial perception..$^{6-9}$ Compared with conventional therapy, computer-assisted cognitive rehabilitation programs have the advantage in that patients can repeat themselves to learn, provide immediate feedback on the performance of patients, and adjust the difficulty level by selecting the appropriate program level for patients.

More recently, studies in the area of cognitive function using three-dimensional virtual reality (VR) programs have been actively conducted. ${ }^{10-13}$ Virtual reality has the advantage of being able to implement the specific circumstance or situation just like a real three-dimensional environment under a virtual environment using a computer and it can be performed regardless of physical disability using the unconstrained interface and minimizing the difference between laboratory environment and real life by interaction with the virtual environment. In particular, recently published studies have reported diagnosis of ambulatory navigational skill delay or reduced ability to perform activities of daily living through the virtual environment or improvement in memory or problem-solving skills through the virtual reality treatment for patients with dementia for whom cognitive decline was observed, ${ }^{14,15}$ patients with Parkinson's disease ${ }^{16}$ or elderly persons with mild cognitive impairment. ${ }^{17,18}$ However, whereas studies of cognitive function using virtual reality for stroke patients are mostly to assess unilateral neglect or overall cognitive impairment using the virtual reality program, ${ }^{19-22}$ there are relatively few studies to prove the effectiveness of virtual reality treatments for cognitive impairment. ${ }^{23}$ In particular, there is little research on the influence on cognitive function when computer-assisted cognitive rehabilitation and virtual reality training are performed at the same time, by comparison with when only computer-assisted cognitive rehabilitation is performed.

The aim of our study was to evaluate the influence on the recovery of cognitive function when the computerassisted cognitive rehabilitation and the virtual reality training are performed together for acute stroke patients with cognitive impairment.

\section{MATERIALS AND METHODS}

\section{Study subjects}

Among patients diagnosed with stroke for the first time by magnetic resonance imaging and computerized tomography from July 1, 2009 to June 30, 2010, we recruited 28 patients who were stabilized neurologically and surgically after undergoing acute phase treatment at the Department of Neurology or Neurosurgery in our hospital and transferred to the Department of Rehabilitation Medicine for comprehensive rehabilitation treatment. All patients had cognitive impairment with a K-MMSE (Korean version of the Mini-Mental Status Examination) score range from 10 to 24 . We excluded patients with a K-MMSE score of less than 10, patients presenting with severe cognitive impairment or aphasia and not being able to understand instructions, patients with poor sitting balance such that they could not sit on a chair with back and armrests, patients with limited range of motion of the neck due to orthopedic problems, and patients with loss of visual acuity such that they could not perceive content on a computer screen. Through random sampling, target patients were classified into two groups. One group was the VR group, which consisted of 15 patients who underwent virtual reality training and computer-assisted cognitive rehabilitation, and the other group was the control group, which consisted of 13 patients who underwent computer-assisted cognitive rehabilitation only. The average ages of the VR and control groups were 66.5 and 62.0 years old, respectively. The average stroke durations from stroke onset to the time of initial evaluation were 18.2 and 24.0 days, respectively. No statistically significant difference was observed in age, sex, duration from stroke onset to the time of initial evaluation, lesion location, number of patients with unilateral neglect, and K-MMSE score 
before treatment of the VR and control groups (Table 1).

\section{Research methods}

The VR group underwent both virtual reality training and computer-assisted cognitive rehabilitation together. Virtual reality training underwent a total of 30 minutes, 3 times a week, and computer-assisted cognitive rehabilitation underwent a total of 30 minutes, 2 times a week for a total of 5 times a week and 4 weeks. The control group underwent computer-assisted cognitive rehabilitation for a total of 30 minutes, 5 times a week, for a total of 4 weeks. All patients were treated with physical therapy and occupational therapy of the same intensity and time duration.

Virtual reality training was performed by one occupational therapist and IREX system ${ }^{\circledR}$ (Vivid group, Toronto, Canada) was used for the virtual reality system. Virtual reality environment consisted of television monitor, video camera, data gloves, and virtual objects (Fig. 1). A video camera recognized the movement or location of a patient and placed them in a virtual reality space, and data gloves recognized the motion of patients responding to virtual reality. We selected 5 out of a total of 20 virtual reality programs for our study. Four different programs were used for one time of virtual reality training, and the programs were correctly set up depending on the patient's condition. Each program was performed in 6 minutes, taking the 2-minute break between programs. Patients were mainly asked to move the affected upper extremity, but were allowed to use the unaffected upper extremity if their motor power was poor grade not being

Table 1. Demographic Characteristics of the Subjects

\begin{tabular}{lcc}
\hline \multicolumn{1}{c}{ Characteristics } & VR group & Control group \\
\hline No. of subjects (N) & 15 & 13 \\
Sex (M/F) & $5 / 10$ & $6 / 7$ \\
Age (year) & $66.5 \pm 11.0$ & $62.0 \pm 15.8$ \\
Day to onset (day) & $18.2 \pm 11.3$ & $24.0 \pm 31.1$ \\
Diagnosis & & \\
Cortical/Subcortical (N) & $10 / 5$ & $8 / 5$ \\
Infarction/Hemorrhage (N) & $12 / 3$ & $9 / 4$ \\
Site of lesion (Rt/Lt) (N) & $9 / 6$ & $8 / 5$ \\
Neglect (N) & 6 & 4 \\
K-MMSE & $17.5 \pm 3.9$ & $16.4 \pm 6.3$ \\
\hline
\end{tabular}

VR: Virtual reality, K-MMSE: Korean version of the Minimental status examination able to lift in themselves against gravity, and sometimes we allowed them to use both upper extremities depending on the program. In addition, since not all patients could be trained while standing, training was performed in the sitting position in a wheelchair. For patients who could not follow the instructions well, it was performed with verbal cues given by the therapist. The first program was called "Bird and Balls", in which balls appeared from various directions on the screen and the patient burst the balls with his/her hands to change them into birds. The second program was called "Coconuts", in which coconut fruits fell down from the top of the screen and the patient put them into a basket by moving his/her arms to the left or right. The third program was called "Conveyor", in which conveyor belts appeared on the left and right sides of the screen and the patient held the box coming from the conveyor belt in one side and moved it to the conveyor belt on the opposite side. The fourth program was called "Juggler", in which one or more balls came up on the screen at the same time, and the patient continued to hit the balls and move them upward to avoid dropping the balls on the ground. The fifth program was called "Soccer", in which a soccer ball came up on the screen and the patient stopped the ball from going in the goal with his/her hands by playing the role of a goalkeeper (Fig. 2). In all programs, the level of difficulty could be controlled by adjusting the velocity, quantity, distance and angle of the object provided by virtual reality de-

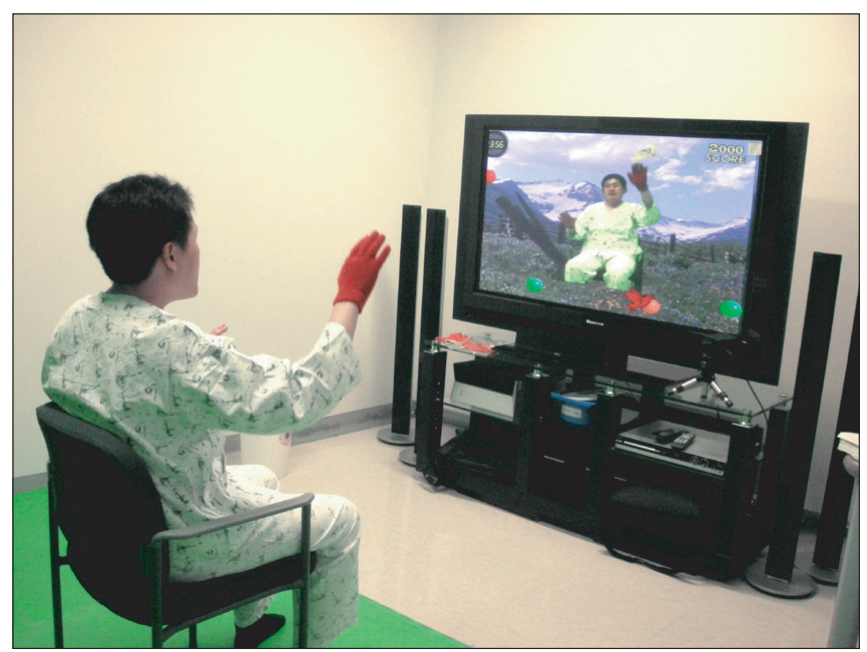

Fig.1. Virtual reality experimental set up of IREX System ${ }^{\circledR}$ (Vivid group, Toronto, Canada). 
pending on the patient's condition. After the end of each program, the screen displayed the score achieved by the patient to make it possible to obtain feedback. In addition, a variety of background music associated with the game was provided in each program.

Computer-assisted cognitive rehabilitation used ComCog ${ }^{\circledR}$ (Maxmedica Inc., Seoul, Korea), which was composed of programs designed to promote attention and memory for patients with cognitive impairment. Attention program was divided into three types of courses, which included the beginner course, intermediate course and advanced course. Based on the complete learning method, the training level could be determined according to the difficulty of the task, and the program details consisted of basic visual perception training, auditory perception training, attention training, attention discrimination training, continuous attention training, attention integration training, and emotion training. Memory programs consisted of three kinds of training courses (beginner, intermediate and advanced) by combining verbal/nonverbal tasks and sequential/non-sequential tasks. It could be classified according to the level of difficulty in the same way as the attention program and the program details consisted of simple recognition memory training, simple spatial memory training, sequential recall memory training, sequential verbal recall memory training, associated recall memory training, verbal categorization memory training, and integrated memory training.

\section{Evaluation methods}

To evaluate cognitive function for all patients before
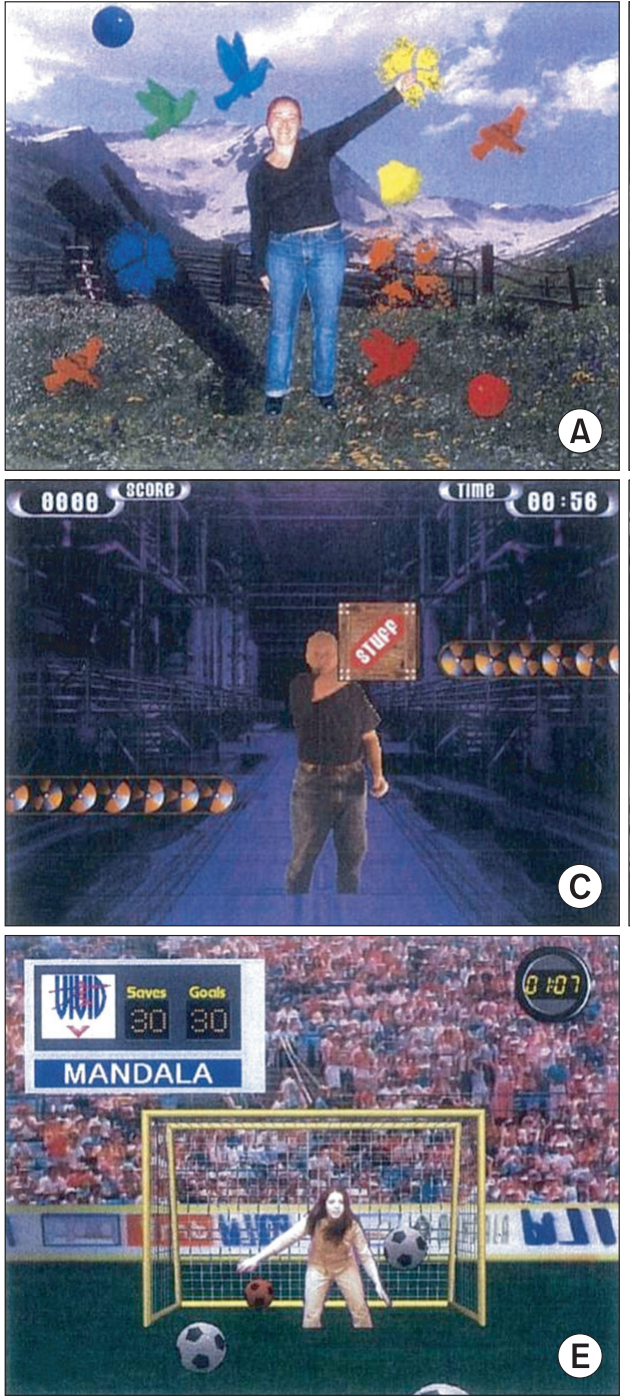

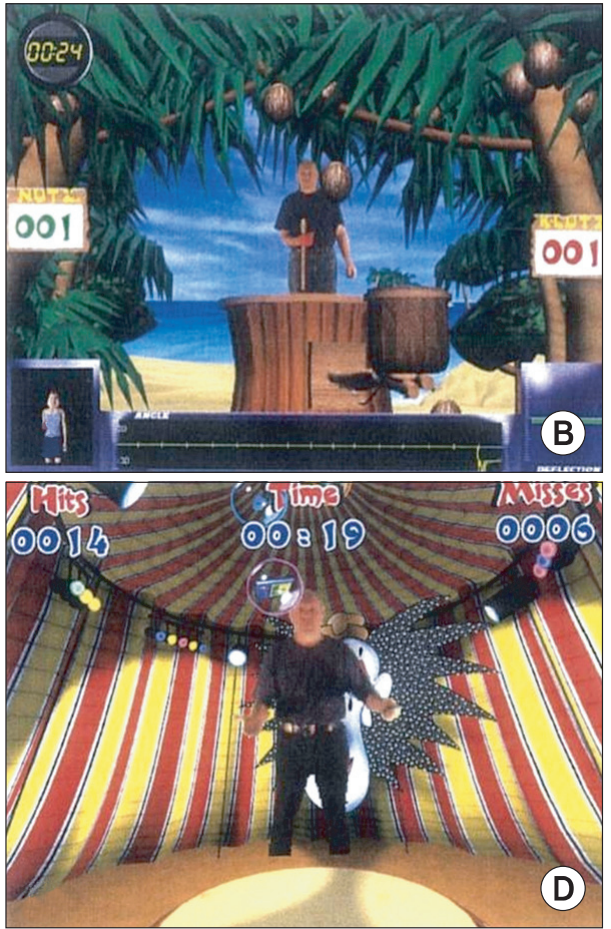

D)

Fig. 2. Five virtual reality programs of IREX system ${ }^{\circledR}$ (Vivid group, Toronto, Canada) (A) Bird and balls (B) Coconutz (C) Conveyor (D) Juggler (E) Soccer. 
treatment, we performed computerized neuropsychological test (CNT, MaxMedica) ${ }^{24}$ and Tower of London test (TOL). ${ }^{25}$

We performed CNT including visual \& auditory continuous performance tests (CPT) to evaluate continuous concentration on visual and auditory stimulations, and word-color test to evaluate the selective attention, and we evaluated forward \& backward digit span tests (DST) and verbal learning test for verbal memory evaluation tests. For the verbal learning test, after letting the patients hear 15 target words through the speaker, we scored the number of words they first recalled, the number of words they recalled after repeating the same target words five times, the number of words they recalled after 20 minutes, and the number of words they found after presenting on the screen a list of 30 words containing the previous 15 words. For the spatial memory test, forward and backward visual span tests (VST) and visual learning tests were performed. For the visual learning test, after presenting 15 different figures sequentially, and then showing 30 figures including 15 figures that were not presented and 15 figures that were previously presented, we scored the number of presented figures they first found, the number of figures they found after showing the same figures five times, the number of figures they found after 20 minutes, and the number of figures they found after showing all 30 figures on the screen. For the visual motor coordination test (Trail making test-type A), we evaluated the time taken to drawing lines connecting 25 small circles on the screen, containing numbers 1 to 25 in each small circle.

For Tower of London (TOL) test to assess executive functioning, 5 balls of different colors were put into each bar among a total of 3 bars and after the occupational therapist showed the final goal position of 5 balls evenly put into 3 bars, we measured the time taken to make the final goal position by moving the balls as little as possible.

We evaluated activities of daily living function using Korean-Modified Barthel Index (K-MBI), and also evaluated motor function using the Motricity Index (MI) for the assessment of upper and lower muscle strength of the affected side after stroke. After 4 weeks of rehabilitation treatment, we re-evaluated the above clinical examination items.

\section{Statistical analysis}

SPSS 14.0 was used for statistical analysis. We analyzed the changes of the described test items including the evaluation of cognitive function before and after treatment within VR and control groups using Wilcoxon signed rank test, and we compared the difference of above test items including the evaluation of cognitive function before and after treatment between the VR and control groups using Mann-Whitney test. For statistical significance, we used 95\% confidence interval, $p<0.05$.

\section{RESULTS}

In the comparison of evaluation items including cognitive function, ability to perform activities of daily living and motor function between the two groups that were tested before treatment, no significant differences between the two groups were observed in all the test items, except for the K-MBI scores $(50.1 \pm 20.3$ vs $30.2 \pm$ 21.6, $\mathrm{p}=0.03$ ), which were significantly higher in the VR group than in the control group.

In the comparison of changes of cognitive function, ability to perform activities of daily living and motor function before and after treatment within the VR and control groups, among the K-MMSE, CNT items, visual and auditory CPTs, forward DST, forward and backward VSTs, and verbal and visual learning tests, TOL test, $\mathrm{K}-\mathrm{MBI}$, and MI scores showed statistically significant improvement in the VR group after treatment when compared with before treatment. In the control group, among the K-MMSE, CNT items, forward DST, visual and verbal learning tests, trail making test-type $\mathrm{A}$, TOL test, K-MBI, and MI scores showed statistically significant improvement after treatment (Table 2).

When comparing the differences of cognitive function, ability to perform activities of daily living and motor function before and after treatment between the VR and control groups, among the CNT items, visual CPT and backward VST showed significant improvement compared to the control group (Table 3).

\section{DISCUSSION}

In our study, we found that visual attention and short-term visuospatial memory showed significant improvement in the VR group compared to the con- 
Table 2. The Changes of Cognitive Activity of Daily Living and Motor Functions before and after Rehabilitation within Each Group

\begin{tabular}{lcccc}
\hline \multirow{2}{*}{ Characteristics } & \multicolumn{2}{c}{ VR group } & \multicolumn{2}{c}{ Control group } \\
\cline { 2 - 5 } & Before & After & Before & After \\
\hline K-MMSE & $17.4 \pm 3.9$ & $21.0 \pm 4.6^{\dagger}$ & $16.4 \pm 6.3$ & $22.1 \pm 5.4^{\dagger}$ \\
\hline VCPT & $0.7 \pm 0.2$ & $0.5 \pm 0.1^{\dagger}$ & $0.6 \pm 0.1$ & $0.5 \pm 0.1$ \\
\hline ACPT & $0.7 \pm 0.1$ & $0.6 \pm 0.1^{*}$ & $0.6 \pm 0.1$ & $0.6 \pm 0.1$ \\
\hline WCW & $38.3 \pm 15.0$ & $34.4 \pm 12.0$ & $26.2 \pm 10.7$ & $23.0 \pm 10.0$ \\
\hline CCW & $67.8 \pm 27.7$ & $68.5 \pm 33.2$ & $69.1 \pm 43.8$ & $46.1 \pm 32.7$ \\
\hline FDST & $4.3 \pm 1.5$ & $4.9 \pm 1.8^{*}$ & $4.5 \pm 1.6$ & $5.3 \pm 1.6^{\dagger}$ \\
BDST & $2.3 \pm 0.5$ & $2.8 \pm 0.9$ & $2.5 \pm 0.8$ & $2,8 \pm 0.8$ \\
\hline FVST & $4.0 \pm 1.0$ & $4.7 \pm 1.3^{*}$ & $3.9 \pm 1.3$ & $4.2 \pm 1.1$ \\
BVST & $3.0 \pm 1.5$ & $3.9 \pm 1.7^{\dagger}$ & $3.1 \pm 1.5$ & $3.0 \pm 1.3$ \\
\hline ViLT- $1^{\text {st }}$ trial & $3.8 \pm 3.7$ & $4.9 \pm 2.5$ & $4.9 \pm 3.8$ & $7.5 \pm 4.4^{*}$ \\
\hline ViLT-5 ${ }^{\text {th }}$ trial & $6.0 \pm 3.6$ & $7.9 \pm 4.3^{*}$ & $6.3 \pm 4.5$ & $6.9 \pm 4.4$ \\
\hline ViLT-recall & $5.6 \pm 3.9$ & $8.9 \pm 3.6^{\dagger}$ & $6.7 \pm 4.0$ & $8.0 \pm 3.8$ \\
\hline ViLT-recognition & $26.1 \pm 13.9$ & $30.8 \pm 12.6^{*}$ & $28.9 \pm 18.9$ & $34.3 \pm 20.2$ \\
\hline VeLT- ${ }^{\text {st }}$ trial & $1.6 \pm 1.6$ & $3.1 \pm 2.2^{\dagger}$ & $2.3 \pm 2.2$ & $3.8 \pm 2.3^{\dagger}$ \\
\hline VeLT-5 ${ }^{\dagger}$ trial & $3.0 \pm 2.5$ & $5.2 \pm 2.4^{*}$ & $4.5 \pm 4.0$ & $6.3 \pm 4.7^{*}$ \\
\hline VeLT-recall & $1.3 \pm 2.5$ & $3.7 \pm 2.9^{*}$ & $2.3 \pm 3.5$ & $4.1 \pm 4.0^{\dagger}$ \\
\hline VeLT-recognition & $12.0 \pm 10.9$ & $19.5 \pm 10.3^{\dagger}$ & $18.8 \pm 14.7$ & $24.4 \pm 16.6^{\dagger}$ \\
\hline TMT-A & $113.9 \pm 72.2$ & $70.9 \pm 44.1$ & $99.1 \pm 54.1$ & $63.1 \pm 44.3^{*}$ \\
\hline TOL & $10.6 \pm 5.9$ & $8.1 \pm 4.2^{\dagger}$ & $8.4 \pm 7.5$ & $6.7 \pm 6.5^{*}$ \\
\hline K-MBI & $50.1 \pm 20.3$ & $69.7 \pm 20.2^{\dagger}$ & $30.2 \pm 21.6$ & $50.9 \pm 25.5^{\dagger}$ \\
\hline MI & $54.6 \pm 24.1$ & $64.0 \pm 26.7^{*}$ & $52.2 \pm 19.8$ & $61.2 \pm 18.5^{*}$ \\
\hline
\end{tabular}

K-MMSE: Korean version of the Mini-mental status examination, VCPT: Visual continuous performance test, ACPT : Auditory continuous performance test, WCW: Word of color word in word-color test, CCW: Color of color word in word-color test, FDST: Forward digit span test, BDST: Backward digit span test, FVST: Forward visual span test, BVST: Backward visual span test, ViLT: Visual learning test, VeLT: Verbal learning test, TMT-A: Trail making test-type A, TOL: Tower of London test, K-MBI: Korean-Modified Barthel index, MI: Motricity index ${ }^{*} \mathrm{p}<0.05,{ }^{\dagger} \mathrm{p}<0.01$

trol group for acute stroke patients with cognitive impairment. Ben-Yishay et al. ${ }^{26}$ reported that intact attention is required to use higher cognitive functions effectively and when the ability to pay attention and concentrate on the information given outside is impaired, memory, problem solving, and production of appropriate actions could be difficult. In other words, they suggested that the loss of attention due to brain injury could interfere with the recovery of other cognitive functions such as memory, executive function, and planning ability. This implies that the cognitive treatment for training and improvement of attention should be based and preceded in order to improve overall cognitive function for braininjured patients with cognitive impairment. In this sense, it is very meaningful that the results of this study showed the improvement of visual attention and short-term visuospatial memory after treatment with the virtual reality program.

Considering the reasons why visual attention and shortterm visuospatial memory significantly improved after virtual reality training: First, virtual reality program was conducted in the forms of the game that could induce motivation and interest of the patients, leading to the improvement of procedural memory and the reactivation of brain neurotransmitters. Therefore, it was likely to improve visual attention and short-term visuospatial memory finally. Rose et al. ${ }^{23}$ reported that among patients with vascular brain injury, the patient group that showed interests in virtual reality environment and actively participated in the program, presented significantly im- 
Table 3. The Comparison of Differences in Cognitive Activity of Daily Living and Motor Functions after Rehabilitation between Two Groups

\begin{tabular}{lcc}
\hline \multicolumn{1}{c}{ Characteristics } & VR group & Control group \\
\hline$\Delta$ K-MMSE & $3.5 \pm 2.8$ & $5.7 \pm 3.3$ \\
$\Delta$ VCPT & $-0.16 \pm 0.19^{\dagger}$ & $-0.03 \pm 0.05$ \\
$\Delta$ ACPT & $-0.06 \pm 0.08$ & $-0.03 \pm 0.08$ \\
$\Delta$ WCW & $-3.9 \pm 16.1$ & $-3.2 \pm 17.2$ \\
$\Delta$ CCW & $0.7 \pm 25.6$ & $-23.0 \pm 34.6$ \\
$\Delta$ FDST & $0.5 \pm 0.8$ & $0.8 \pm 0.7$ \\
$\Delta$ BDST & $0.4 \pm 0.9$ & $0.3 \pm 0.8$ \\
$\Delta$ FVST & $0.7 \pm 1.0$ & $0.3 \pm 1.1$ \\
$\Delta$ BVST & $0.9 \pm 0.8^{*}$ & $-0.1 \pm 1.0$ \\
$\Delta$ ViLT- ${ }^{\text {st }}$ trial & $1.2 \pm 2.8$ & $2.6 \pm 3.7$ \\
$\Delta$ ViLT- $5^{\text {th }}$ trial & $1.9 \pm 2.5$ & $0.6 \pm 2.5$ \\
$\Delta$ ViLT-recall & $3.3 \pm 3.0$ & $1.3 \pm 2.3$ \\
$\Delta$ ViLT-recognition & $4.8 \pm 6.1$ & $5.4 \pm 14.3$ \\
$\Delta$ VeLT- $1^{\text {st }}$ trial & $1.5 \pm 1.3$ & $1.6 \pm 1.4$ \\
$\Delta$ VeLT- $5^{\text {th }}$ trial & $2.2 \pm 2.4$ & $1.8 \pm 1.9$ \\
$\Delta$ VeLT-recall & $2.4 \pm 2.6$ & $1.8 \pm 1.7$ \\
$\Delta$ VeLT-recognition & $6.9 \pm 6.9$ & $5.6 \pm 6.3$ \\
$\Delta$ TMT-A & $-43.0 \pm 83.6$ & $-36.0 \pm 42.7$ \\
$\Delta$ TOL & $-2.5 \pm 2.0$ & $-1.7 \pm 2.3$ \\
$\Delta$ K-MBI & $19.6 \pm 17.8$ & $20.8 \pm 10.1$ \\
$\Delta$ MI & $9.4 \pm 11.2$ & $8.6 \pm 8.3$ \\
\hline
\end{tabular}

K-MMSE: Korean version of the Mini-mental status examination, VCPT: Visual continuous performance test, ACPT: Auditory continuous performance test, WCW: Word of color word in word-color test, CCW: Color of color word in word-color test, FDST: Forward digit span test, BDST: Backward digit span test, FVST: Forward visual span test, BVST: Backward visual span test, ViLT: Visual learning test, VeLT: Verbal learning test, TMT-A: Trail making test-type A, TOL: Tower of London test, K-MBI: Korean-Modified Barthel index, MI: Motricity index

${ }^{*} \mathrm{p}<0.05,{ }^{\dagger} \mathrm{p}<0.01$

proved spatial awareness test scores, compared to the patient group who watched the program passively. Rose et al. ${ }^{23}$ explained that since there were many cases in which procedural memory was preserved even if there was brain injury, procedural memory would have been helpful to improve visuospatial memory during the active participation and devotion in virtual reality program. In addition, Kim et al. ${ }^{27}$ reported that if the patients were motivated by experiencing a diverse and stimulating environment through the virtual reality program, it would be able to result in the improvement of attention and memory by the reactivation of brain neurotransmitter capability such as cholinergic and dopaminergic systems when combined with cognitive treatment. Second, the screen backgrounds for the virtual reality used in our study were a natural environment such as mountains and fields, skiing resorts, soccer grounds suitable for each program, and by placing the patients in the stimulusrich virtual backgrounds for visuospatial perception, their visuospatial perception abilities were likely to be improved. Several studies ${ }^{28,29}$ demonstrated that visuospatial learning ability improved significantly when treated with stimulus training of visuospatial perception through the virtual reality program in the form of game for patients with physical disability, compared with the patients not treated with virtual reality program. Third, it might be able to improve the patient's level of alertness and visual attention during the treatment by providing continuous visual stimulus through the dynamic screen along with various auditory stimulus such as background music offered in the virtual reality program. In fact, we found that visual attention as well as auditory attention showed significant improvement within the VR group before and after treatment. Kim et al. ${ }^{27}$ reported the results of attention and memory performance improvement after performing virtual reality program of cognitive function for the elderly. The previous study proposed an explainable mechanism that strong virtual reality stimulus (visual and auditory) and immersiveness enough to induce the user active cognitive activities have been involved in improvement of cognitive function.

In addition, our study showed significant improvement in verbal and visual learning tests reflecting relatively long-term memory as well as short-term memory and attention before and after treatment in the VR group. Patients in the VR group performed simple and repetitive motions primarily using shoulder flexion and abduction through the virtual reality program, and repetitive physical movements in such virtual reality was likely to improve cognitive function by increasing the activation of the brain responsible for cognitive functions. Fordyce and Farrar ${ }^{30}$ reported that continued treadmill exercise of rats brought improvements in visuospatial memory. Grealy et al. ${ }^{31}$ observed in their study that when various visual stimulus were given to patients with traumatic 
brain injury by experiencing bicycle riding by themselves in virtual reality, they showed significant improvement in digit symbol tests as well as visual and verbal learning tests. Grealy et al. ${ }^{31}$ suggested that an interactive exercise could be associated with improvement of functions in the hippocampal region and a relatively simple physical exercise could result in the improvement of learning ability by reducing the overload of attention through virtual reality. However, because the control group showed significant improvement in one item of visual learning test and verbal learning test, it may be difficult to conclude simply that it was therapeutic effects of virtual reality. In addition, a TOL test to assess planning ability showed significant improvement in both groups before and after treatment, and there are limitations to conclude that it was the effect of virtual reality only. In order to prove its effectiveness, it is considered necessary to compare the group treated only with virtual reality training and the group treated only with computer-assisted cognitive rehabilitation.

In our study, we did not observe significant improvement of ability to perform activities of daily living in patients treated with virtual reality, compared to other patients. We considered that repetitive movements of the shoulder are required in the virtual reality programs, but making fine motor movements of hand such as catching and moving things are usually required in the activities of daily living. Therefore, it may cause limitations that lead to functional improvement. In addition, the improvement in activities of daily living may not appear significant because there are many cases which need other cognitive functions such as planning ability, problem solving ability and working memory in everyday life.

Limitations of our study included not observing significant differences in the number of patients showing unilateral neglect between both groups before treatment. Nonetheless, we have not determined whether the improvement of visual attention and short-term visuospatial memory correlated with the improvement of unilateral neglect because we did not investigate whether the unilateral neglect improved after treatment. Of course, several studies ${ }^{22,32,33}$ reported the improvement of unilateral neglect through virtual reality training, but the virtual reality programs used in those studies reproduced the situations representing unilateral neglect by letting patients cross the virtual street or to respond to objects that appear in the direction of occurring unilateral neglect by using only the upper extremity. Thus, those programs are different from the contents of virtual reality program that is conducted in the form of game as shown in our study. Therefore, further research is needed to investigate the improvement of unilateral neglect by using the virtual reality programs used in our study. Second, as mentioned earlier, we failed to clarify the effectiveness of virtual reality treatment for longterm visual and verbal memory since the comparison with the group underwent virtual reality treatment only was not performed. However, because computerassisted cognitive rehabilitation has been used widely for cognitive treatment of stroke patients with cognitive impairment, we could not perform only virtual reality treatment for these patients. Finally, we could not conduct a comparative study on the effectiveness of virtual reality treatment depending on lesion location of cerebral hemisphere, cerebral cortical or subcortical areas due to the small number of patients.

\section{CONCLUSION}

In our study, we found that stroke patients with cognitive impairment showed significant improvement of visual attention and short-term visuospatial memory when treated with computer-assisted cognitive rehabilitation and virtual reality training together, compared to the cases when treated with computer-assisted cognitive rehabilitation only. Thus, in clinical practice, the additional effect is expected for the improvement of cognitive function when treated with virtual reality therapy together with computer-assisted cognitive rehabilitation therapy for cognitive rehabilitation of stroke patients with cognitive impairment. In the future, further efforts will be needed to develop virtual reality programs and demonstrate their effectiveness for improvement in various cognitive areas including verbal memory, problem-solving ability, and planning ability as well as attention and short-term visuospatial memory.

\section{REFERENCES}

1. Leys D, Henon H, Pasquier F. White matter changes and poststroke dementia. Dement Geriatr Cogn Disord 1998; 9 Suppl 1: 25-29 
2. Pohjasvaara T, Erkinjuntti T, Vataja R, Kaste M. Dementia three months after stroke. Baseline frequency and effect of different definitions of dementia in the Helsinki Stroke Aging Memory Study (SAM) cohort. Stroke 1997; 28: 785-792

3. Cicerone KD, Dahlberg C, Kalmar K, Langenbahn DM, Malec JF, Berquist TF, Felicetti T, Giacino JT, Harley JP, Harrington DE, et al. Evidence-based cognitive rehabilitation: recommendations for clinical practice. Arch Phys Med Rehabil 2000; 81: 1596-1615

4. Gontkovsky ST, McDonald NB, Clark PG, RuWe WD. Current directions in computer-assisted cognitive rehabilitation. Neurorehabilitation 2002; 17: 195-199

5. Diamond PT, Felsenthal G, Maccinocchi SN, Butler DH, Lally-Cassady D. Effect of cognitive impairment on rehabilitation outcome. Am J Phys Med Rehabil 1996; 75: 40-43

6. Glisky EL, Schacter DL, Tulving E. Learning and retention of compter-related vocabulary in memoryimpaired patients: method of vanishing cues. J Clin Exp Neuropsychologia 1986; 8: 292-312

7. Chen SH, Thomas JD, Glueckauf RL, Bracy OL. The effectiveness of computer-assisted cognitive rehabilitation for persons with traumatic brain injury. Brain injury 1997; 11: 197-209

8. Niemann H, Ruff RM, Base CA. Computer-assisted attention retraining in head-injured individuals: a controlled efficacy study of an outpatient program. J Consult Clin Psychol 1990; 58: 811-817

9. Kim YH, Ko MH, Seo JH, Park SH, Kim KS, Jang EH, Park SW, Park JH, Cho YJ. Effect of computer-assisted cognitive rehabilitation program for attention training in brain injury. J Korean Acad Rehab Med 2003; 27 : 830-839

10. Parsons TD, Rizzo AA. Initial validation of a virtual environment for assessment of memory functioning: virtual reality cognitive performance assessment test. Cyberpsychol Behav 2008; 11: 17-25

11. Parsons TD, Rizzo AA. Neurpsychological assessment using the virtual reality cognitive performance assessment test. Proceedings of the 7th ICDVRAT with ArtAbilitation; 2008 Aug 31-Sep 2; Maia, Portugal. UK: University of Reading; 2008

12. Parsons TD, Larson P, Kratz K, Thiebaux M, Bluestein B, Buckwalter JG, Rizzo AA. Sex differences in mental rotation and spatial rotation in a virtual environment.
Neuropsychologia 2004; 42: 555-562

13. Matheis RJ, Schultheis MT, Tiersky LA, Deluca J, Millis SR, Rizzo A. Is learning and memory different in a virtual environment? Clin Neuropsychol 2007; 21: 146-161

14. Hofmann M, Rosler A, Schwarz W, Muller-Spahn F, Krauchi K, Hock C, Seifritz E. Interactive computertraining as a therapeutic tool in Alzheimer's disease. Compr Psychiatry 2003; 44: 213-219

15. Cushman LA, Stein K, Duffy CJ. Detecting navigational deficits in cognitive aging and Alzheimer disease using virtual reality. Neurology 2008; 71: 888-895

16. Davidsdottir S, Wagenaar R, Young D, Cronin-Golomb A. Impact of optic flow perception and egocentric coordinates on veering in Parkinson's disease. Brain 2008; 131: 2882-2893

17. Werner P, Rabinowitz S, Klinger E, Korczyn AD, Josman N. Use of the virtual action planning supermarket for the diagnosis of mild cognitive impairment: a preliminary study. Dement Geriatr Cogn Disord 2009; 27: 301-309

18. Optale G, Urgesi C, Busato V, Marin S, Piron L, Priftis K, Gamberini L, Capodieci S, Bordin A. Controlling memory impairment in elderly adults using virtual reality memory training: a randomized controlled pilot study. Neurorehabil Neural Repair 2010; 24: 348357

19. Kim DY, Joo SY, Park CI, Park TH, Par KD, Jung KJ, Lee JH. Assessment of post-stroke cognitive dysfunction using 3-dimensional virtual reality program. J Korean Acad Rehab Med 2009; 33: 12-20

20. Kim DY, Lee JH, Park CI, Kim YW, Chang WH, Kim IY, Kim SI, Chon JS, Chang HJ. Assessment of post-stroke unilateral neglect using 3-dimensional virtual reality program. J Korean Acad Rehab Med 2005; 29: 1-8

21. Kang YJ, Ku JG, Han KW, Kim SI, Yu TW, Lee JH, Park CI. Development and clinical trial of virtual realitybased cognitive assessment in people with stroke: preliminary study. Cyberpsychol Behav 2008; 11: 329339

22. Kim JH, Kim KG, Kim DY, Chang WH, Park CI, Ohn SH, Han KW, Ku JH, Nam SW, Kim IY, et al. Virtual environment training system for rehabilitation of stroke patients with unilateral neglect: crossing the virtual street. Cyberpsychol Behav 2007; 10: 7-15

23. Rose FD, Brooks BM, Attree EA, Parslow DM, Lead- 
better AG, McNaill JE, Jayawardena S, Greenwood $\mathrm{R}$, Potter J. A preliminary investigation into the use of virtual environments in memory retraining after vascular brain injury: indications for future strategy? Disabil Rehabil 1999; 21: 548-554

24. Kim YH, Shin SH, Park SH, Ko MH. Cognitive assessment for patient with brain injury by computerized neuropsychological test. J Korean Acad Rehab Med 2001; 25: 209-216

25. Phillips LH, Wynn V, Gilhooly KJ, Della Sala S, Logie RH. The role of memory in the tower of London task. Memory 1999; 7: 209-231

26. Ben-Yishay Y, Piasetsky EB, Rattock J. A systematic method for ameliorating disorders in basic attention. In: Meier MJ, Benton AL, Diller L, Editors. Neuropsychological rehabilitation, 1st ed, New York, Churchill Livingstone, 1987, 165-181

27. Kim MY, Lee KS, Choi JS, Kim HB, Park CI. Effectiveness of cognitive training based on virtual reality for the elderly. J Korean Acad Rehab Med 2005; 29: 424433

28. Wilson PN, Foreman N, Tlauka M. Transfer of spatial information from a virtual to a real environment in physically disabled children. Disabil Rehabil 1996; 18: 633-637

29. Stanton D, Foreman N, Wilson P, Duffy H, Parnell R. Use of virtual environments to acquire spatial understanding of real-world multi-level environments. Proceedings of the 4th International Conference on Disability, Virtual Reality and associated Technologies; 2002 Sep 18-20; Veszprem, Hungary. UK: University of Reading; 2002

30. Fordyce DE, Farrar RP. Enhancement of spatial learning in F344 rats by physical activity and related learning-associated alterations in hippocampal and cortical cholinergic functioning. Behav Brain Res 1991; 46: 123-133

31. Grealy MA, Johnson DA, Rushton SK. Improving cognitive function after brain injury: the use of exercise and virtual reality. Arch Phys Med Rehabil 1999; 80: 661-667

32. Castiello U, Lusher D, Burton C, Glover S, Disler P. Improving left hemispatial neglect using virtual reality. Neurology 2004; 62: 1958-1962

33. Glover S, Castiello U. Recovering space in unilateral neglect: a neurological dissociation revealed by virtual reality. J Cogn Neurosci 2006; 18: 833-843 